\title{
EFECTO DE LA INTELIGENCIA SOBRE EL FAKING EN UNA MEDIDA DE PERSONALIDAD EN CONTEXTO DE SELECCIÓN DE PERSONAL: UN ESTUDIO DE DIF
}

\section{EFFECT OF INTELLIGENCE ON FAKING IN A PERSONALITY MEASURE IN THE CONTEXT OF PERSONNEL SELECTION: A DIF STUDY}

Martínez Ordóñez, G. y Luzdivina Fisteus, M.

Universidad Complutense de Madrid

\section{RESUMEN}

Introducción: Una de las críticas a la utilización de autoinformes de personalidad en contextos de selección es la posibilidad de falseamiento de las respuestas o "Faking”. Algunos han propuesto que esta habilidad podría reflejar diferencias individuales en inteligencia. El objetivo de este estudio es determinar si diferencias observadas en personalidad son diferencias verdaderas en el constructo, o si están contaminadas por un sesgo cognitivo. Método: Se han considerado dos grupos: uno de alto CI (116-130) $(\mathrm{N}=1004)$ y otro de bajo CI (70-85) ( $\mathrm{N}=996)$. Media de edad en torno a 26 años. Se realizaron análisis DIF (Funcionamiento Diferencial de los Ítems) mediante: Regresión logística, Estandarización y SIBTEST. Resultados: 1) Existen diferencias significativas en las dimensiones de personalidad del modelo Big Five; 2) Se ha detectado una elevada proporción de ítems con DIF; 3) Las diferencias entre grupos se reducen significativamente al considerar ítems sin DIF. Discusión: Las implicaciones serán discutidas.

Palabras claves: Selección de personal, Faking, autoinformes, personalidad, inteligencia, DIF. 


\section{SUMMARY}

Introduction: One of the criticisms at the use of personality self-reports in selection contexts is the possibility of Faking. Some authors have suggested that this ability may reflect individual differences in intelligence. The aim of this study is to figure out whether differences observed in personality are true in the construct or seem corrupted by a cognitive bias. Method: It has been considered two groups: one of high IQ (116-130) $(\mathrm{N}=1004)$ and one of low IQ (70-85) ( $\mathrm{N=996).} \mathrm{Age} \mathrm{average} \mathrm{around} \mathrm{26.} \mathrm{DIF} \mathrm{(Differential} \mathrm{Functioning} \mathrm{of}$ Items) analysis were made using: Logistical regression, Standardization and SIBTEST. Results: 1) Significant differences in all dimensions of personality from Big Five model; 2) It has been detected a high amount of items with DIF; 3) When non-DIF items are taken into account, differences between groups reduce significantly. Discussion: present results implications will be discussed.

Key words: Staff selection, Faking, self-reports, personality, intelligence and DIF.

\section{INTRODUCCIÓN}

El conocimiento de las relaciones entre la personalidad y el desempeño laboral ha supuesto un avance significativo dentro del ámbito de la Psicología del trabajo (Táuriz, 2011). Las medidas de personalidad son buenos predictores de algunos criterios organizacionales relevantes (e.g. Barrick y Mount, 1991). Sin embargo, a pesar de su éxito para predecir el desempeño su utilización no está exenta de problemas (Salgado, 2005).

Una de las críticas más reiteradas es la posibilidad de falseamiento de las respuestas (“Facking”). Éste se ha definido como la tendencia a contestar a los ítems de modo que se responde a las presiones sociales (Ellingson, Smith y Sackett, 2001 citado en Táuriz 2011). Esta distorsión puede ser tanto de signo positivo (Deseabilidad Social), como de signo negativo. La primera, que implica sobredimensionar las actitudes y comportamientos socialmente deseables y reducir aquéllos desaprobados (Jiménez \& Domínguez-Espinosa, 2010). Está formada por dos aspectos: “manejo de impresión” (intención) y “autoengaño” (no intencionado) (Paulhus, 1991). La deseabilidad tiende a aumentar las puntuaciones en las dimensiones relacionadas positivamente con el desempeño y a disminuir las relacionadas negativamente (Salgado, 2005). Este falseamiento supone una amenaza a la validez y, por tanto, a las decisiones de contratación (véase e.g. Douglas, McDaniel, y Snell, 1996).

Algunos autores han propuesto que la habilidad para el "fake" podría reflejar diferencias individuales en inteligencia (e.g. García et al., 2006). Para abordar el estudio de la 
influencia de la inteligencia sobre la evaluación de la personalidad en contextos de selección, emplearemos una aproximación basada en el DIF, considerando dos grupos con puntuaciones extremas en inteligencia. Cuando la probabilidad de responder correctamente a un ítem difiere de un grupo a otro por factores ajenos al propio constructo se produce Funcionamiento Diferencial del Ítem (DIF). Aquí se analizaran los dos tipos de DIF posibles: uniforme y no uniforme (Abad, Olea, Ponsada \& García, 2011).

\section{MÉTODO \\ Participantes}

Se contó con una muestra procedente de un gran proceso de selección para una empresa multinacional de transportes (participación de 18932 personas), extrayéndose dos grupos: uno de alto CI (116-130) $(\mathrm{N}=1004,50.7 \%$ mujeres) y otro de bajo $\mathrm{CI}(70-85)(\mathrm{N}=996,50.6 \%$ varones). La media de edad estuvo en torno a los 26 años (DT=5).

\section{Instrumentos}

Se aplicó un cuestionario de personalidad de 150 ítems, creado por la empresa a partir del banco disponible en la web “International Personality Item Pool”, siguiendo el modelo Big Five. La medida de inteligencia empleada para formar los grupos fue el Test de Matrices Progresivas de Raven, en su escala avanzada.

\section{Procedimiento y Análisis de datos}

Los participantes fueron evaluados y entrevistados en distintas ciudades de España. El trabajo de campo fue realizado por los técnicos de Recursos Humanos de la empresa entre octubre-febrero de 2014/2015. Los cuestionarios se administraron en grupos. Se les pasó un consentimiento informado y a continuación, se administró el Raven y la prueba de personalidad. Los datos fueron grabados en una única base de datos informatizada (marzoabril de 2015). Para el análisis se empleó una aproximación basada en DIF mediante: regresión logística, estandarización y SIBTEST.

\section{RESULTADOS}

En primer lugar, se analizaron las puntuaciones promedio (tabla 1) para las cinco dimensiones del modelo Big Five, obteniéndose tamaños del efecto entre 0.73 y 1.07. 
Tabla 1. Puntuaciones promedio

\begin{tabular}{l|ccccc}
\hline \multirow{2}{*}{} & \multicolumn{2}{c}{ ALTO CI } & \multicolumn{2}{c}{ BAJO CI } & \multirow{2}{*}{ d } \\
\cline { 2 - 5 } Nedia & D.T & Media & D.T & 1.07 \\
\hline $\mathbf{N} \_N D I F$ & 39.47 & 11.09 & 50.63 & 9.81 & .54 \\
\hline $\mathbf{E}$ & 19.90 & 6.64 & 23.45 & 6.49 & .73 \\
\hline E_NDIF & 80.25 & 9.12 & 73.74 & 8.82 & .51 \\
\hline O & 39.42 & 5.56 & 36.60 & 5.49 & .77 \\
\hline O_NDIF & 73.15 & 7.95 & 66.97 & 8.05 & .50 \\
\hline A & 42.57 & 5.14 & 39.97 & 5.26 & .87 \\
\hline A_NDIF & 84.02 & 8.84 & 77.04 & 7.13 & .42 \\
\hline R & 37.07 & 4.43 & 35.20 & 4.40 & .99 \\
\hline R_NDIF & 90.02 & 7.34 & 81.73 & 9.33 & .62 \\
\hline
\end{tabular}

En la tabla 2 se presentan ítems detectados por los diferentes métodos DIF:

- Regresión Logística: determina si en la función matemática necesaria para predecir las respuestas del ítem se debe incluir el nivel de habilidad o el grupo de pertenencia o la interacción entre ambos.

- Estandarización: solo detecta DIF uniforme.

- SIBTEST: permite determinar si los ítems con DIF provocarán un fenómeno de amplificación o de cancelación.

Tabla 2. Ítems detectados por los diferentes métodos DIF y decisión final basada en la comparación entre métodos.

\begin{tabular}{|c|c|c|c|c|c|c|c|}
\hline & \multicolumn{2}{|c|}{$\begin{array}{l}\text { REGRESIÓN } \\
\text { LOGÍSTICA }\end{array}$} & \multicolumn{2}{|c|}{ ESTANDARIZACIÓN } & \multicolumn{2}{|c|}{ SIBTEST } & \multirow{2}{*}{$\begin{array}{l}\text { DECISIÓN } \\
\text { FINAL }\end{array}$} \\
\hline & DIF U & DIF NoU & ALTO CI & BAJO CI & ALTO CI & BAJO CI & \\
\hline $\mathbf{N}$ & $13(43 \%)$ & $2(7 \%)$ & $4(13 \%)$ & $10(33 \%)$ & $4(13 \%)$ & $9(30 \%)$ & $15(50 \%)$ \\
\hline $\mathbf{E}$ & $12(40 \%)$ & $3(10 \%)$ & $9(30 \%)$ & $6(20 \%)$ & $9(30 \%)$ & $6(20 \%)$ & $15(50 \%)$ \\
\hline $\mathbf{O}$ & $10(33 \%)$ & $3(10 \%)$ & $8(27 \%)$ & $5(17 \%)$ & $7(23 \%)$ & $4(13 \%)$ & $12(40 \%)$ \\
\hline A & $14(47 \%)$ & $3(10 \%)$ & $10(33 \%)$ & $5(17 \%)$ & $10(33 \%)$ & $5(17 \%)$ & $16(53 \%)$ \\
\hline $\mathbf{R}$ & $16(53 \%)$ & $2(7 \%)$ & $12(40 \%)$ & $5(17 \%)$ & $12(40 \%)$ & $4(13 \%)$ & $17(57 \%)$ \\
\hline
\end{tabular}

Como se observa en la tabla 2, para el modelo de Regresión Logística existen 78 ítems que presentan DIF, 65 de ellos uniforme; es decir, que la probabilidad de contestar correctamente a un ítem es mayor para el grupo de alto CI en todos los niveles de rasgo. En la Estandarización se advierte un mayor número de ítems con DIF en el grupo de alto CI para el rasgo Responsabilidad (40\%), mientras que para el grupo focal, los ítems que presentan mayor DIF uniforme son los de Neuroticismo. El SIBTEST muestra mayor DIF en Responsabilidad (40\%) para el grupo de alto CI, así como mayor DIF para los de bajo CI en Neuroticismo. Además, solo se produce fenómeno de amplificación al comparar subtest sospechosos de DIF y el subtest válido. 
Por último, la decisión final de los ítems que presentan DIF en el test de personalidad, basada en la comparación entre métodos, determina la presencia predominante de DIF para el rasgo de Responsabilidad. Apertura a la Experiencia, rasgo que menos DIF presenta (40\%).

\section{DISCUSIÓN Y CONCLUSIONES}

El propósito del presente estudio ha sido determinar si las diferencias observadas en personalidad en un contexto de selección son verdaderas en el constructo, o están contaminadas por un sesgo de capacidad cognitiva. Los resultados muestran diferencias significativas en las cinco dimensiones de personalidad del Big Five.

Se ha detectado una elevada proporción de ítems con DIF en la dirección de lo que se podría denominar un perfil socialmente deseable. Abad et al. (2011) han mostrado que este hecho indica que la respuesta depende de una variable externa al constructo evaluado pero que influye en él. Cuando estos ítems con DIF son eliminados, las diferencias entre grupos se reducen significativamente.

Los resultados sugieren que el nivel de CI es un potente moderador de las tendencias de Faking. Así pues, candidatos con mayor puntuación en el test de inteligencia responderían de forma más sesgada y directa a los ítems de las dimensiones de Responsabilidad y Amabilidad, mientras que tenderían a mostrar el sesgo inverso en los ítems de Neuroticismo.

Futuras investigaciones deberían profundizar acerca del alcance del fenómeno estudiado. Asimismo, se deberían poner a prueba hipótesis causales acerca de las razones que subyacen a la presencia del DIF. Sería de gran interés poder analizarlo utilizando cuestionarios de personalidad estándar: por ejemplo el Big Five (Caprara, Borgogni, Barbaranelli \& Moreno, 1995).

\section{Agradecimientos}

Las autoras agradecen al profesor/Dr. Sergio Escorial Martín del Departamento de Metodología de las Ciencias del Comportamiento, de la Facultad de Psicología, Universidad Complutense de Madrid por la revisión cuidadosa que ha realizado de este texto y sus valiosas aportaciones. 


\section{BIBLIOGRAFÍA}

Abad, F. J., Olea, J., Ponsada, V. \& García, C. (2011). Medición en Ciencias Sociales y de la Salud. Madrid: Síntesis.

Barrick, M.R. y Mount, M.K. (1991). The Big Five personality dimensions and job performance: A meta-analysis. Personnel Psychology, 44, 1-26.

Caprara, G. V., Borgogni, L., Barbaranelli, C., \& Moreno, J. B. (1995). BFQ: Cuestionario " Big Five": Manual. Madrid : TEA Ediciones.

Douglas, E. F., McDaniel, M. A. y Snell, A. F. (1996). The validity of non-cognitive measures decays when applicants fake. Academy of Management Proceedings, 6, 127131.

García, L.F., Cuevas, L., Escorial, S., Aluja, A., De Fruyt, F., Rolland, J.P. (2006, Julio). Differential Item Functioning of NEO-PI-R Items as a Function of Intelligence in Personnel Selection Assessment. Poster presentado en 13th European Conference on Personality. Atenas (Grecia).

Jiménez, F. J., \& Domínguez-Espinosa, A. C. (2010). Influencia de la Deseabilidad Social en Reportes de Capacitación. Psicología Iberoamericana, 18(1), 69-79.

Paulhus, D. L. (1991). Measurement and control of response bias. In J. P. Robinson, P. R. Shaver \& L. S. Wrightsman (Eds.), Measure of personality and social psychological attitudes (pp. 17-59). San Diego, CA: Academic Press.

Salgado, J. F. (2005). Personalidad y deseabilidad social en contextos organizacionales: implicaciones para la práctica de la psicología del trabajo y las organizaciones. Papeles de Psicólogos, 26, 115-128.

Táuriz, G. (2011). Falseamiento y validez de las medidas de personalidad en contextos académicos. Revista de Psicología del Trabajo y de las Organizaciones, 27(2), 103115.

Ellingson, J. E., Smith, D. B. y Sackett, P. R. (2001). Investigating the influence of social desirability on personality factor structure. Journal of Applied Psychology, 86, 122133. 